\title{
Mechanisms of Nephronophthisis and Related Ciliopathies
}

\author{
Toby W. Hurd ${ }^{\mathrm{b}}$ Friedhelm Hildebrandt $\mathrm{t}^{\mathrm{a}, \mathrm{b}}$ \\ ${ }^{a}$ Howard Hughes Medical Institute and ${ }^{b}$ Departments of Pediatrics and Human Genetics, University of \\ Michigan, Ann Arbor, Mich., USA
}

\section{Key Words}

Nephronophthisis - NPHP genes · Joubert syndrome •

Meckel-Gruber syndrome $\cdot$ Senior-Løken syndrome

\begin{abstract}
An emerging group of human genetic diseases termed 'ciliopathies' are caused by dysfunction of two functionally and physically associated organelles, the centrosome and cilium. These organelles are central to perception of the physical environment through detection of a diverse variety of extracellular signals such as growth factors, chemicals, light and fluid flow. Many of the described ciliopathies display multiorgan involvement, with renal and retina being the most commonly affected. Nephronophthisis is a recessive disorder of the kidney that is the leading cause of end-stage renal failure in children. Through positional cloning, many of the causative mutations have been mapped to genes involved in centrosome and cilia function. In this review, we discuss the identified causative mutations that give rise to nephronophthisis and how these are related to the disease etiology in both the kidney and other organs.
\end{abstract}

Copyright $\odot 2010$ S. Karger AG, Basel

\section{KARGER}

Fax +4161306 1234

E-Mail karger@karger.ch

www.karger.com
(C) 2010 S. Karger AG, Basel

Accessible online at: www.karger.com/nee

\section{Cilia, Centrosomes and Ciliopathies}

In the area of developmental cell biology, intense scrutiny has been focused on understanding the formation and function of cilia and centrosomes, in part driven by positional cloning of human disease genes and the discovery that they are expressed at cilia and centrosomes [Hildebrandt and Otto, 2005]. Cilia are microtubulebased organelles that are nucleated from the mother centriole of the centrosome in quiescent cells and protrude into the extracellular environment. Through cell typespecific specialization and compartmentalization of the proteins within the cilium, they are ideally suited for detection of various extracellular stimuli including light, fluid flow, growth factors and chemicals.

Remarkably, the core set of proteins required to form cilia, the intraflagellar transport (IFT) proteins, are highly conserved through evolution from the bi-flagellate green algae Chlamydomonas reinhardtii up to humans. Subsequently, it has been demonstrated that there is also astonishing evolutionary conservation of human ciliopathy proteins, such as those mutated in polycystic kidney disease (PKD), nephronophthisis (NPHP) and BardetBiedl syndrome (BBS). This evolutionary conservation has been central to the rapid growth of our understanding of the etiology of these diseases through the use of model organisms including C. reinhardtii, Caenorhabditis elegans, zebrafish and mice. Driving this field was the

Friedhelm Hildebrandt, MD

Howard Hughes Medical Institute, Departments of Pediatrics and Human Genetics University of Michigan Health System, 8220C MSRB III

1150 West Medical Center Drive, Ann Arbor, MI 48109-5646 (USA)

Tel. +1 734615 7285, Fax +1 734615 1386, E-Mail fhilde@ umich.edu 
Table 1. Genes mutated in nephronophthisis and related ciliopathies

\begin{tabular}{lllll}
\hline Locus & Gene & Protein & Functional domains & Associated syndrome \\
\hline NPHP1 & NPHP1 & nephrocystin 1 & CC, SH3 & NPHP, SLS \\
NPHP2 & $I N V$ & inversin & ANK, IQ & NPHP, SLS \\
NPHP3 & NPHP3 & nephrocystin 3 & CC, TPR & NPHP, SLS \\
NPHP4 & NPHP4 & nephroretinin & - & NPHP, SLS \\
NPHP5 & IQCB1 & IQ motif containing B1 & CC, IQ & SLS \\
NPHP6 & CEP290 & centrosomal protein 290 & CC & NPHP, SLS, JS, MKS \\
NPHP7 & GLIS2 & GLI-similar 2 & ZF & NPHP, SLS, JS, MKS \\
NPHP8 & RPGRP1L & RPGRIP1-like & CC, C2 & NPHP, SLS \\
NPHP9 & NEK8 & NIMA-related kinase 8 & STK & SLS, BBS-like \\
NPHP10 & SDCCAG8 & serologically defined colon cancer antigen 8 & CC & NPHP, JS, MKS, LF \\
NPHP11 & TMEM67 & transmembrane protein 67 & NPHP \\
NPHPL1 & XPNPEP3 & X-prolyl aminopeptidase 3 & peptidase & JS \\
JBTS1 & INPP5E & inositol polyphosphate-5-phosphatase & IPP & TM \\
JBTS2 & TMEM216 & transmembrane protein 216 & JC, WD40, SH3 \\
JBTS3 & AHI1 & Jouberin & GTPase, CC & JS, MKS \\
JBTS8 & ARL13B & ADP-ribosylation factor-like 13B & JS \\
JBTS9 & CC2D2A & coiled coil and C2 domain containing 2A & CC & JS, MKS \\
MKS1 & MKS1 & Meckel-Gruber syndrome type 1 & - & MKS \\
\hline
\end{tabular}

CC = Coiled coil; SH3 = Src homology 3; ANK = ankyrin repeat; IQ = isoleucine glutamine motif; TPR = tetratricopeptide repeat; $\mathrm{ZF}=$ zinc finger; $\mathrm{C} 2=\mathrm{Ca}^{2+}$-binding motif; $\mathrm{STK}=$ serine threonine kinase; $\mathrm{TM}=$ transmembrane; $\mathrm{IPP}=$ inositol polyphosphate phosphatase; $\mathrm{WD} 40=\beta$-transducin repeat; $\mathrm{LF}=$ liver fibrosis.

intial observation that mutation of the IFT protein IFT88/ polaris in the $\mathrm{Tg} 737$ mouse model of autosomal dominant PKD (ADPKD) led to shortened cilia in the kidney [Pazour et al., 2000]. Subsequently, it was established that the polypeptides encoded by genes mutated in human ADPKD such as polycystin 2 localized to primary cilia in renal epithelia [Yoder et al., 2002]. To date, more than thirty different genes have been identified that, when mutated, give rise to renal cystic cilia-related disease (ciliopathies), and the majority of these localize to cilia and/or centrosomes. It is also becoming evident that many of these genes encode proteins that physically interact with other ciliopathy proteins to form large macromolecular complexes required for correct cilia function. This has been nicely demonstrated for the ciliopathy BBS, where proteomic analysis revealed a complex consisting of the BBS1, BBS2, BBS4, BBS5, BBS7, BBS8 and BBS9 proteins [Nachury et al., 2007]. In this review, we will focus on the ciliopathy NPHP.

\section{Nephronophthisis}

NPHP is an autosomal recessive kidney disease that is the most common cause of inheritable end-stage renal failure (ESRF) in the first three decades of life, with the median onset of ESRF being at 13 years. The disease can be subdivided clinically based on the age of onset of ESRF into infantile, juvenile and adolescent categories with the median age of onset being 1, 13 and 19 years of age, respectively. Prior to ESRF, clinical symptoms include polyuria, polydipsia and anemia. Kidneys from NPHP patients are generally normal or reduced in size. Histologically, they display sporadic cysts at the corticomedullary junction, tubular basement membrane disruption, periglomerular fibrosis and tubulointerstitial cell infiltrates with interstitial fibrosis.

\section{NPHP1 Deletions Cause a Limited Disease}

To date, through the use of positional cloning, mutations in twelve genes (NPHP1-11 and NPHPL1) have been demonstrated to give rise to NPHP (table 1). However, these genes only account for approximately $30 \%$ of cases of NPHP. So it is likely that many more causative genes will be identified in the future. The first identified NPHP gene was NPHP1 that encodes the protein nephrocystin-1, which is mutated in approximately $20 \%$ of all cases of NPHP. Homozygous deletions of the NPHP1 gene were identified in individuals with juvenile NPHP type 1 
[Hildebrandt et al., 1997]. This protein was first described to localize to epithelial cell-cell contacts [Donaldson et al., 2000, 2002], but was later additionally localized to the cilia transition zone [Fliegauf et al., 2006]. Interestingly, this localization requires the interaction of NPHP1 with the trafficking protein PACS1 which is also involved in the trafficking of the ADPKD protein polycystin 2 [Kottgen et al., 2005; Schermer et al., 2005].

\section{NPHP2 Mutations Cause Infantile NPHP}

The NPHP2 gene encodes the inversin protein and is mutated in infantile NPHP [Otto et al., 2003]. Unusually, unlike the other NPHP genes, individuals harboring mutations in inversin have slightly enlarged kidneys that more closely resemble kidneys from PKD patients. In addition, patients may display situs inversus and cardiac ventricular septal defects. Like nephrocystin-1, inversin also localizes to the proximal part of the cilium [Shiba et al., 2009]. Of all the NPHP genes, the causative disease mechanism associated with inversin is the best understood. Pioneering work by Simons et al. [2005] showed that cilia are essential for the regulation of planar cell polarity and that inversin plays a central role in this process. Inversin acts at a fulcrum between the Wnt-mediated canonical and noncanonical pathways. Loss of inversin function through its mutation results in enhanced noncanonical Wnt signaling and abrogated planar cell polarity. Planar cell polarity specifies the orientation of a cell with regards to neighboring cells and is essential for normal tissue formation and maintenance. It is thought that cyst formation, especially in $\mathrm{PKD}$, results from randomized orientation of epithelial cell division that leads to ductal expansion [Fischer et al., 2006; Verdeguer et al., 2010].

\section{Mutations of NPHP3, -4 and -5 Cause Retinal-Renal Ciliopathies}

Mutations of the NPHP3 gene which encodes nephrocystin 3 have been found in patients with adolescent NPHP [Olbrich et al., 2003; Tory et al., 2009]. A missense mutation of Nphp3 also causes the renal cystic mouse phenotype $P C Y$ [Olbrich et al., 2003]. Similar to inversin, nephrocystin 3 localizes to the proximal region of primary cilia, and this localization requires its interaction with inversin [Shiba et al., 2010]. In addition, nephrocystin 3 also forms a complex with nephrocystin 1 . The function of nephrocystin 3 is not clear, but it is fundamental to cilia function as truncating mutations of NPHP3 in both humans and mice result in extremely severe multiorgan dysfunction as a result of embryonic patterning defects which closely resemble those observed in MeckelGruber syndrome (MKS) [Bergmann et al., 2008].

The nephrocystin 4 protein encoded by the NPHP4 gene, which is mutated in juvenile NPHP, localizes to the cilia transition zone as well as to the cortical actin cytoskeleton of epithelia [Mollet et al., 2005; Winkelbauer et al., 2005]. It is thought that NPHP4 in conjunction with NPHP1 may function at the transition zone to regulate entry and exit of ciliary cargos [Winkelbauer et al., 2005]. More recently, NPHP4, again in conjunction with NPHP1, was deemed essential in regulating cellular apicobasal polarity via interactions with the evolutionarily conserved PALS1/PATJ/Crb3 polarity complex [Delous et al., 2009]. Apicobasal polarity of epithelia is essential for formation of cell-cell contacts known as tight junctions which prevent paracellular movement of molecules across epithelia, as well as for cilia formation. However, it is not clear whether the primary etiology of NPHP is due to abnormal cell polarity or cilia dysfunction. NPHP4 has also been demonstrated to interact with two other ciliopathy proteins, RPGRIP and RPGRIP1L, which are mutated in Leber congenital amaurosis and cerebello-oculo-renal syndrome (Joubert syndrome, JS) respectively. Mutations in RPGRIP1L were found to give rise to NPHP [Arts et al., 2007; Delous et al., 2009].

Similar to NPHP4, the NPHP5 gene product IQCB1 also localizes to primary cilia. In addition, it interacts with the retinal ciliopathy gene RPGR (retinitis pigmentosa GTPase regulator) which is mutated in the majority of cases of X-linked retinitis pigmentosa [Otto et al., 2005]. IQCB1 contains a calmodulin-binding IQ domain, and does in fact directly interact with calmodulin. However, the functional significance of this interaction is not clear. Although renal cilia regulate intracellular calcium levels in response to fluid flow, it is likely that calcium and calmodulin regulate many aspects of cilia formation and function.

Since mutation of any one NPHP gene closely recapitulates the phenotype of mutations in other NPHP genes, together with the fact that most of these proteins are localized to cilia and centrosomes, it is highly likely that the nephrocystin proteins form supramolecular complexes that are necessary for cilia formation and function. Indeed, the IQCB1 protein directly interacts with CEP290/NPHP6 [Schafer et al., 2008]. This is further supported by the fact that like NPHP5, NPHP6 also forms a complex with RPGR [Chang et al., 2006]. 
NPHP6, -7 and -8 Implicate Planar Cell Polarity, Hedgehog Signaling and Cell Cycle Regulation

An additional level of complexity was revealed by the direct interaction and subsequent activation of NPHP6 with the cAMP-regulated transcription factor CREB2/ ATF4 [Sayer et al., 2006]. It has been known for some time that elevated cAMP levels are observed in epithelia from cystic kidneys [Wang et al., 2010], and this observation was the first to provide evidence that abnormal gene expression may contribute to disease progression of NPHP. Knockdown of Cep290/NPHP6 in zebrafish recapitulated the JS phenotype seen in humans and demonstrated a planar cell polarity phenotype.

A signaling mechanism that was more recently demonstrated to be linked to primary cilia is the hedgehog pathway. Hedgehog signaling is crucial during embryogenesis as it controls tissue patterning and cell fate specification. The hedgehog receptor Patched localizes to primary cilia, and upon hedgehog binding subsequently traffics out of the cilia allowing the protein Smoothened (Smo) to reside in the cilium [May et al., 2005; Ocbina and Anderson, 2008]. Ciliary Smo then promotes the conversion of Gli transcription factors, which also localize to cilia, to the activator forms that when trafficked to the nucleus drive expression of hedgehog responsive genes. In the case of NPHP, a related transcription factor Glisimilar 2 was found to be mutated in NPHP type 7 [Attanasio et al., 2007]. Mutation of the NPHP7 locus in mice resulted in many of the hallmark histological features of NPHP such as renal atrophy and prominent fibrosis [Attanasio et al., 2007]. Loss of Gli-similar 2 resulted in a transcriptional switch that led to upregulation of genes that promote epithelial to mesenchymal transition, potentially providing an explanation for the fibrosis associated with NPHP. This observation together with the association of NPHP6 and ATF4 again highlighted the central role that NPHP proteins may play in maintenance of normal kidney function through the regulation of gene expression.

Although PKD and NPHP have markedly different histological features, the gene products involved share a common subcellular distribution - the cilia and centrosomes. A further connection was established upon the identification of the NPHP9 locus. The NPHP9 gene encodes the kinase NEK8 which localizes to the proximal part of the cilium. NPHP causative mutations resulted in mislocalization of NEK from this region [Otto et al., 2008]. In a subsequent study, it was found that NEK8 interacts with the ADPKD protein polycystin 2 and sug- gested that NEK8 may regulate both the expression and posttranslational modification (phosphorylation) of both polycystin 1 and polycystin 2 [Sohara et al., 2008]. It has also been shown that NPHP2, which when mutated gives a PKD-like kidney phenotype, is required for targeting of NEK8 to the primary cilium [Shiba et al., 2010]. It is likely that more such connections involving the cilia-centrosome complex will be subsequently found between genes involved in the hyperplastic phenotype of PKD and the dysplastic phenotype of NPHP.

Most recently, we have demonstrated that not all NPHP genes localize to primary cilia or centrosomes. Using genome-wide homozygosity mapping, we identified a new NPHP locus in two families with NPHP-like symptoms. The NPHP-like 1 gene (NPHPL1) encodes the enzyme X-prolyl aminopeptidase 3 (XPNPEP3) which, contrary to the current ciliopathy paradigm, localizes to mitochondria via a mitochondrial leader sequence. However, whilst XPNPEP3 does not localize to cilia, it may modulate cilia function through proteolytic cleavage of a number of cilia proteins harboring compatible proteolytic target motifs [O’Toole et al., 2010].

\section{Extrarenal Phenotypes and Allelism}

As previously discussed, cilia are highly conserved organelles whose function may be modified by the incorporation of additional tissue-specific proteins that modulate their cell type-specific function. This requirement for the same core protein complexes that underlie cilia function between different cell types results in syndromic ciliopathies, where multiple ciliated tissues are effected by mutation of a single gene. This is especially true of nephropthisis, which is often associated with extrarenal manifestations.

\section{Senior-Løken Syndrome}

One of the most common extrarenal manifestations associated with NPHP is retinal degeneration. Both rod and cone photoreceptors have specialized cilium that act to connect the inner and outer segments. Initially during retinal morphogenesis, photoreceptor cells contain a single primary cilium that closely resembles the one seen on many other cell types. However, the cilia membrane of photoreceptors becomes highly specialized through delivery of material to and expansion of the ciliary distal tip. Compared to the cilia of other cell types, the photoreceptor 
connecting cilium likely experiences particularly high traffic due to turnover of rhodopsin and light-dark adaptation. As such, photoreceptors are likely to be overtly sensitive to mutations that effect cilia function/trafficking. In fact, nephronophthisis is often associated with retinal degeneration due to mutation of cilia-associated proteins. Senior-Løken syndrome (SLS) is a renal-retinal disorder caused by mutations in the several of the NPHP genes. All patients with mutations in the NPHP5 gene and approximately $30 \%$ of patients with mutations in NPHP4 or NPHP9 exhibit SLS. Recently, we have also identified mutations in a new gene NPHP10/SDCCAG8. Like many of the other NPHP genes, SDCCAG8 is a centrosomal-associated protein which directly interacts with the ciliopathy protein OFD1. Most patients with NPHP10 mutations exhibit SLS, but a few additionally display some features of BBS such as obesity and mild mental retardation. [Otto et al., 2010].

\section{Joubert Syndrome and Meckel-Gruber Syndrome}

Similar to SLS, JS results in renal-retinal manifestations but with accompanied cerebellar vermis hypoplasia. JS patients therefore exhibit multiple neurological defects such as ataxia and mental retardation. JS can be caused by recessive mutations in each of multiple different NPHP genes including NPHP1, NPHP3, NPHP6 and NPHP8. A further syndromic ciliopathy with multi-organ involvement is MKS. MKS is a recessive disorder that results in prenatal lethality due to multiple organ dyspla- sia including kidney (kidney cysts), retina (microphthalmia), brain (occipital meningoencephalocele), liver (hepatic cysts) and limbs (postaxial polydactyly). Of the NPHP genes, MKS can be caused by recessive mutations in either NPHP3, NPHP6 or NPHP8.

It is becoming clear that mutations in a specific NPHP gene do not always correlate with a specific genotype/ phenotype. Mutations in one gene such as NPHP5/ CEP290 can give rise to a broad spectrum of phenotypes from NPHP with no extrarenal manifestations through to JS or MKS. The severity of the phenotype may belinked to the type of mutations/alleles present, such that severe mutations (truncating/null) would give rise to severe disease (such as MKS) and hypomorphic (missense) alleles give rise to milder disease (NPHP). This alleism effect may also be relevant to disease progression, where severe alleles result in an early-onset phenotype due to developmental defects (organ formation/patterning) and milder alleles give rise to later-onset degenerative defects (apoptosis/fibrosis). With the advent of high-throughput sequencing techniques, it is likely that the list of causative genes and mutations will continue to grow.

\section{Acknowledgements}

This work was supported by grants from the National Institutes of Health to F.H. (DK1069274, DK1068306, DK064614). F.H. is an Investigator of the Howard Hughes Medical Institute, a Doris Duke Distinguished Clinical Scientist and a Frederick G.L. Huetwell Professor.

\section{References}

Arts HH, Doherty D, van Beersum SE, Parisi MA, Letteboer SJ, Gorden NT, Peters TA, Marker T, Voesenek K, Kartono A, et al: Mutations in the gene encoding the basal body protein RPGRIP1L, a nephrocystin- 4 interactor, cause Joubert syndrome. Nat Genet 2007;39:882-888.

-Attanasio M, Uhlenhaut NH, Sousa VH, O’Toole JF, Otto E, Anlag K, Klugmann C, Treier AC, Helou J, Sayer JA, et al: Loss of GLIS2 causes nephronophthisis in humans and mice by increased apoptosis and fibrosis. Nat Genet 2007;39:1018-1024.

-Bergmann C, Fliegauf M, Bruchle NO, Frank V, Olbrich H, Kirschner J, Schermer B, Schmedding I, Kispert A, Kranzlin B, et al: Loss of nephrocystin-3 function can cause embryonic lethality, Meckel-Gruber-like syndrome, situs inversus, and renal-hepaticpancreatic dysplasia. Am J Hum Genet 2008; 82:959-970.
Chang B, Khanna H, Hawes N, Jimeno D, He S, Lillo C, Parapuram SK, Cheng H, Scott A, Hurd RE, et al: In-frame deletion in a novel centrosomal/ciliary protein CEP290/ NPHP6 perturbs its interaction with RPGR and results in early-onset retinal degeneration in the rd16 mouse. Hum Mol Genet 2006;15:1847-1857.

Delous M, Hellman NE, Gaude HM, Silbermann F, Le Bivic A, Salomon R, Antignac C, Saunier S: Nephrocystin-1 and nephrocystin-4 are required for epithelial morphogenesis and associate with PALS1/PATJ and Par6. Hum Mol Genet 2009;18:4711-4723.

Donaldson JC, Dempsey PJ, Reddy S, Bouton AH, Coffey RJ, Hanks SK: Crk-associated substrate p130(Cas) interacts with nephrocystin and both proteins localize to cell-cell contacts of polarized epithelial cells. Exp Cell Res 2000;256:168-178.
Donaldson JC, Dise RS, Ritchie MD, Hanks SK: Nephrocystin-conserved domains involved in targeting to epithelial cell-cell junctions, interaction with filamins, and establishing cell polarity. J Biol Chem 2002;277:2902829035.

Fischer E, Legue E, Doyen A, Nato F, Nicolas JF, Torres V, Yaniv M, Pontoglio M: Defective planar cell polarity in polycystic kidney disease. Nat Genet 2006;38:21-23.

- Fliegauf M, Horvath J, von Schnakenburg C, Olbrich H, Muller D, Thumfart J, Schermer B, Pazour GJ, Neumann HP, Zentgraf H, et al: Nephrocystin specifically localizes to the transition zone of renal and respiratory cilia and photoreceptor connecting cilia. J Am Soc Nephrol 2006; 17:2424-2433.

Hildebrandt F, Otto E: Cilia and centrosomes: a unifying pathogenic concept for cystic kidney disease? Nat Rev Genet 2005;6:928-940. 
-Hildebrandt F, Otto E, Rensing C, Nothwang HG, Vollmer M, Adolphs J, Hanusch H, Brandis M: A novel gene encoding an $\mathrm{SH} 3$ domain protein is mutated in nephronophthisis type 1. Nat Genet 1997;17:149153.

Kottgen M, Benzing T, Simmen T, Tauber R, Buchholz B, Feliciangeli S, Huber TB, Schermer B, Kramer-Zucker A, Hopker K, et al: Trafficking of TRPP2 by PACS proteins represents a novel mechanism of ion channel regulation. EMBO J 2005;24:705-716.

-May SR, Ashique AM, Karlen M, Wang B, Shen Y, Zarbalis K, Reiter J, Ericson J, Peterson AS: Loss of the retrograde motor for IFT disrupts localization of Smo to cilia and prevents the expression of both activator and repressor functions of Gli. Dev Biol 2005;287:378-389.

- Mollet G, Silbermann F, Delous M, Salomon R, Antignac C, Saunier S: Characterization of the nephrocystin/nephrocystin-4 complex and subcellular localization of nephrocystin- 4 to primary cilia and centrosomes. Hum Mol Genet 2005;14:645-656.

- Nachury MV, Loktev AV, Zhang Q, Westlake CJ, Peranen J, Merdes A, Slusarski DC, Scheller RH, Bazan JF, Sheffield VC, Jackson PK: A core complex of BBS proteins cooperates with the GTPase Rab8 to promote ciliary membrane biogenesis. Cell 2007;129:1201-1213.

-O'Toole JF, Liu Y, Davis EE, Westlake CJ, Attanasio M, Otto EA, Seelow D, Nurnberg G, Becker $C$, Nuutinen $M$, et al: Individuals with mutations in XPNPEP3, which encodes a mitochondrial protein, develop a nephronophthisis-like nephropathy. J Clin Invest 2010;120:791-802.

- Ocbina PJ, Anderson KV: Intraflagellar transport, cilia, and mammalian Hedgehog signaling: analysis in mouse embryonic fibroblasts. Dev Dyn 2008;237:2030-2038.

- Olbrich H, Fliegauf M, Hoefele J, Kispert A, Otto E, Volz A, Wolf MT, Sasmaz G, Trauer U, Reinhardt R, et al: Mutations in a novel gene, NPHP3, cause adolescent nephronophthisis, tapeto-retinal degeneration and hepatic fibrosis. Nat Genet 2003;34:455-459. $\checkmark$ Otto EA, Hurd TW, Airik R, et al: Candidate exome capture identifies mutation of SDCCAG8 as the cause of a retinal-renal ciliopathy. Nat Genet 2010;42:840-850.

Otto EA, Loeys B, Khanna H, Hellemans J, Sudbrak R, Fan S, Muerb U, O’Toole JF, Helou J, Attanasio M, et al: Nephrocystin-5, a ciliary IQ domain protein, is mutated in Senior-Loken syndrome and interacts with RPGR and calmodulin. Nat Genet 2005;37:282-288.

Otto EA, Schermer B, Obara T, O’Toole JF, Hiller KS, Mueller AM, Ruf RG, Hoefele J, Beekmann F, Landau D, et al: Mutations in INVS encoding inversin cause nephronophthisis type 2, linking renal cystic disease to the function of primary cilia and left-right axis determination. Nat Genet 2003;34:413-420.

Otto EA, Trapp ML, Schultheiss UT, Helou J, Quarmby LM, Hildebrandt F: NEK8 mutations affect ciliary and centrosomal localization and may cause nephronophthisis. J Am Soc Nephrol 2008; 19:587-592.

- Pazour GJ, Dickert BL, Vucica Y, Seeley ES, Rosenbaum JL, Witman GB, Cole DG: Chlamydomonas IFT88 and its mouse homologue, polycystic kidney disease gene $\operatorname{tg} 737$, are required for assembly of cilia and flagella. J Cell Biol 2000;151:709-718.

- Sayer JA, Otto EA, O’Toole JF, Nurnberg G, Kennedy MA, Becker C, Hennies HC, Helou J, Attanasio M, Fausett BV, et al: The centrosomal protein nephrocystin- 6 is mutated in Joubert syndrome and activates transcription factor ATF4. Nat Genet 2006;38:674681.

Schafer T, Putz M, Lienkamp S, Ganner A, Bergbreiter A, Ramachandran H, Gieloff V, Gerner M, Mattonet C, Czarnecki PG, et al: Genetic and physical interaction between the NPHP5 and NPHP6 gene products. Hum Mol Genet 2008;17:3655-3662.

Schermer B, Hopker K, Omran H, Ghenoiu C, Fliegauf M, Fekete A, Horvath J, Kottgen M, Hackl M, Zschiedrich S, et al: Phosphorylation by casein kinase 2 induces PACS-1 binding of nephrocystin and targeting to cilia. EMBO J 2005;24:4415-4424.
Shiba D, Manning DK, Koga H, Beier DR, Yokoyama T: Inv acts as a molecular anchor for Nphp3 and Nek8 in the proximal segment of primary cilia. Cytoskeleton 2010;67:112119.

- Shiba D, Yamaoka Y, Hagiwara H, Takamatsu T, Hamada H, Yokoyama T: Localization of Inv in a distinctive intraciliary compartment requires the C-terminal ninein-homolog-containing region. J Cell Sci 2009;122:44-54.

-Simons M, Gloy J, Ganner A, Bullerkotte A, Bashkurov M, Kronig C, Schermer B, Benzing T, Cabello OA, Jenny A, et al: Inversin, the gene product mutated in nephronophthisis type II, functions as a molecular switch between Wnt signaling pathways. Nat Genet 2005;37:537-543.

Sohara E, Luo Y, Zhang J, Manning DK, Beier DR, Zhou J: Nek8 regulates the expression and localization of polycystin-1 and polycystin-2. J Am Soc Nephrol 2008;19:469-476.

- Tory K, Rousset-Rouviere C, Gubler MC, Moriniere V, Pawtowski A, Becker C, Guyot C, Gie S, Frishberg Y, Nivet H, et al: Mutations of NPHP2 and NPHP3 in infantile nephronophthisis. Kidney Int 2009;75:839-847.

- Verdeguer F, Le Corre S, Fischer E, Callens C, Garbay S, Doyen A, Igarashi P, Terzi F, Pontoglio M: A mitotic transcriptional switch in polycystic kidney disease. Nat Med 2010;16: 106-110.

-Wang X, Ward CJ, Harris PC, Torres VE: Cyclic nucleotide signaling in polycystic kidney disease. Kidney Int 2010;77:129-140.

-Winkelbauer ME, Schafer JC, Haycraft CJ, Swoboda P, Yoder BK: The C. elegans homologs of nephrocystin-1 and nephrocystin-4 are cilia transition zone proteins involved in chemosensory perception. J Cell Sci 2005; 118:5575-5587.

-Yoder BK, Hou X, Guay-Woodford LM: The polycystic kidney disease proteins, polycystin-1, polycystin-2, polaris, and cystin, are co-localized in renal cilia. J Am Soc Nephrol 2002;13:2508-2516. 